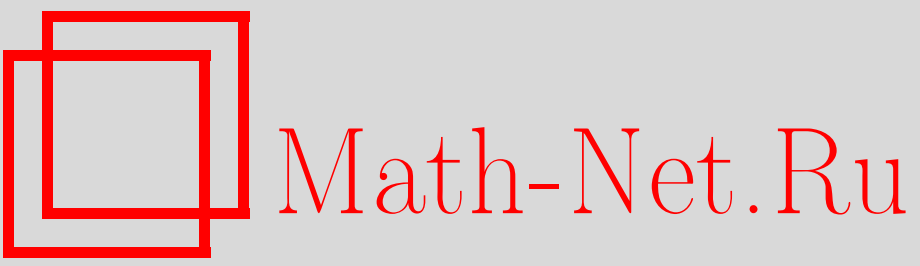

Л. А. Бассалыго, В. А. Зиновьев, Многочлены специального вида над конечным полем с максимальным модулем тригонометрической суммы, УМН, 1997, том 52, выпуск 2, 31-44

DOI: https://doi.org/10.4213/rm816

Использование Общероссийского математического портала Math-Net.Ru подразумевает, что вы прочитали и согласны с пользовательским соглашением

http://www.mathnet.ru/rus/agreement

Параметры загрузки:

IP: 3.91 .87 .62

26 апреля 2023 г., 07:26:06 


\section{МНОГОЧЛЕНЫ СПЕЦИАЛЬНОГО ВИДА НАД \\ КОНЕЧНЫМ ПОЛЕМ С МАКСИМАЛЬНЫМ МОДУЛЕМ ТРИГОНОМЕТРИЧЕСКОЙ СУММЫ}

Л. А. БАССАЛЫГО, В.А. ЗИНОВЬЕВ

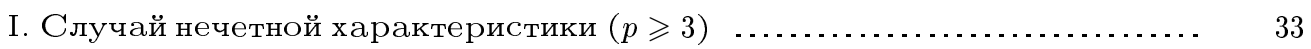

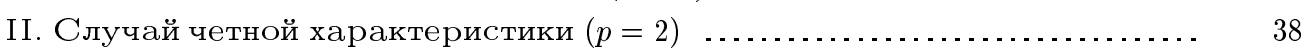

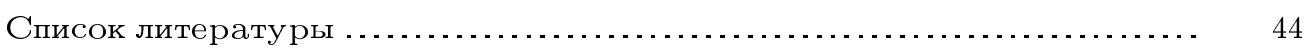

Пусть $q=p^{l}, Q=q^{m}=p^{l m}$, где $p$ - простое число, $l, m$ - целые положительные числа, $m>1, F_{p} \subseteq F_{q} \subset F_{Q}$ - соответствуюшие конечные поля. Пусть, далее,

$$
\begin{gathered}
f(x)=a_{1} x+\cdots+a_{n} x^{n}, \quad a_{i} \in F_{Q}, \\
S(f)=\sum_{x \in F_{Q}} \exp \left\{\frac{2 \pi i \operatorname{Tr}_{Q}(f(x))}{p}\right\}
\end{gathered}
$$

где

$$
\operatorname{Tr}_{Q}(x)=x+x^{p}+x^{p^{2}}+\cdots+x^{p^{l m-1}}, \quad x \in F_{Q} .
$$

Нам понадобится также представление $\operatorname{Tr}_{Q}(x)$ в виде

$$
\operatorname{Tr}_{Q}(x)=\operatorname{Tr}_{q}\left(\operatorname{Tr}_{Q \rightarrow q}(x)\right),
$$

где

$$
\operatorname{Tr}_{Q \rightarrow q}(x)=x+x^{q}+\cdots+x^{q^{m-1}}, \quad x \in F_{Q} .
$$

В настоящей работе мы изучаем значение модулей тригонометрических сумм для многочленов специального вида:

$$
f(x)=\sum_{s=0}^{\lfloor m / 2\rfloor} a_{s} x^{1+q^{s}}, \quad a_{s} \in F_{Q}, \quad \operatorname{Tr}_{Q}(f(x)) \neq \text { const }
$$

(т.е. мы исключаем тривиальньй случай, когда $|S(f)|=Q$ ). Исследование многочленов несколько более общего вида

$$
f^{\prime}(x)=\sum_{i, j=0}^{m-1} a_{i j} x^{q^{i}+q^{j}}
$$


не добавляет новых значений тригонометрических сумм, ибо из соотношения $\operatorname{Tr}_{Q \rightarrow q}(y)=\operatorname{Tr}_{Q \rightarrow q}\left(y^{q}\right), y \in F_{Q}$, вытекает, что для любого многочлена $f^{\prime}(x)$ найдется многочлен $f(x)$ вида (3) такой, что

$$
\operatorname{Tr}_{Q \rightarrow q} f^{\prime}(x)=\operatorname{Tr}_{Q \rightarrow q} f(x)
$$

и, следовательно, $S\left(f^{\prime}\right)=S(f)$.

Вообще исследование тригонометрических сумм многочлена от одной переменной над полем $F_{q^{m}}$ сводится к исследованию кратных тригонометрических сумм многочлена от $m$ переменных над полем $F_{q}$. Действительно, пусть $\Phi(x)$ - произвольньй многочлен над полем $F_{Q}, Q=q^{m}$. Зафиксируем базис $\gamma_{0}, \gamma_{1}, \ldots, \gamma_{m-1}$ поля $F_{Q}$ над полем $F_{q}$ : любой элемент $x \in F_{Q}$ представим в виде

$$
x=\sum_{s=0}^{m-1} x_{s} \gamma_{s}, \quad x_{s} \in F_{q} .
$$

Положим

$$
G\left(x_{0}, x_{1}, \ldots, x_{m-1}\right)=\operatorname{Tr}_{Q \rightarrow q} \Phi\left(\sum_{s=0}^{m-1} x_{s} \gamma_{s}\right)
$$

Ясно, что $G\left(x_{0}, \ldots, x_{m-1}\right)$ - многочлен от $m$ переменных над полем $F_{q}$. По определению кратной тригонометрической суммы

$$
S_{m}\left(G\left(x_{0}, \ldots, x_{m-1}\right)\right)=\sum_{x_{0}, \ldots, x_{m-1} \in F_{q}} \exp \left\{\frac{2 \pi i \operatorname{Tr}_{q}\left(G\left(x_{0}, \ldots, x_{m-1}\right)\right)}{p}\right\}
$$

и в силу $(1),(2),(4)$ имеем, что

$$
S(\Phi(x))=S_{m}\left(G\left(x_{0}, \ldots, x_{m-1}\right)\right) .
$$

Нахождение по многочлену $G\left(x_{0}, \ldots, x_{m-1}\right)$ такого многочлена $\Phi(x)$, что вьполнено равенство (4), также возможно (этому была посвяшена работа [1]). Конечно, такое сведение помогает лишш в том случае, когда мы можем выгислить кратную тригонометрическую сумму. Один такой случай - это квадратичные формы [2], [3]. В работе [1] мы показали, что для любой квадратичной формы $g\left(x_{0}, \ldots, x_{m-1}\right)$ соответствующий многочлен $\Phi(x)$ (см. (4)) может быть выбран в виде (3). С другой стороны, нетрудно видеть, что для любого многочлена $f(x)$ вида (3) соответствуюший многочлен $G\left(x_{0}, \ldots, x_{m-1}\right)$ (см. (4)) является квадратичной формой $g\left(x_{0}, \ldots, x_{m-1}\right)$. Известно [2], [3], что модуль тригонометрической суммы квадратичных форм от $m$ переменных над полем $F_{q}$ может принимать любое значение:

$$
\begin{aligned}
q^{r / 2}, \text { где } r & =2 m-1,2 m-2, \ldots, m, q \text {-нечетное }, \\
q^{r}, \text { где } r & =m-1, m-2, \ldots,\lceil m / 2\rceil, q \text {-четное },
\end{aligned}
$$

и никаких других ненулевых нетривиальных $\left(\neq q^{m}\right)$ значений принимать не может. Следовательно, то же самое относится и к модулю тригонометрической суммы многочленов вида (3). В данной работе описаны многочлены вида (3) с максимальным значением модуля тригонометрической суммы и приведено много примеров многочленов, для которых достигается граница Вейля: $|S(f)| \leqslant(n-1) \sqrt{Q}$. 


\section{I. Случай нечетной характеристики $(p \geqslant 3)$}

В этом случае максимальное значение модуля тригонометрической суммы многочленов вида (3) равно $q^{m-\frac{1}{2}}$ [1] (для нечетных $m$ это значение совпадает с границей Вейля). С.А. Степанов [4] дал примеры таких многочленов в явном виде. Здесь мы описьваем все такие многочлены.

Для нечетного $q$ любой многочлен вида (3) может быть представлен в следующем виде:

$$
\varphi(x)=a_{0} x^{2}+2 \sum_{s=1}^{\lfloor m / 2\rfloor} a_{s} x^{1+q^{s}} .
$$

Для нахождения коэффициентов соответствующей (см. (4)) квадратичной формы

$$
g\left(x_{0}, \ldots, x_{m-1}\right)=\operatorname{Tr}_{Q \rightarrow q}\left(\varphi\left(\sum_{s=0}^{m-1} x_{s} \gamma_{s}\right)\right)=\sum_{u, v=0}^{m-1} c_{u v} x_{u} x_{v}, \quad c_{u v}=c_{v u}
$$

вьпишем вначале $\operatorname{Tr}_{Q \rightarrow q}(\varphi(x))$ :

$$
\operatorname{Tr}_{Q \rightarrow q}(\varphi(x))=\sum_{i, j=0}^{m-1} b_{i j} x^{q^{i}+q^{j}}
$$

где

$$
\begin{gathered}
b_{i i}=a_{0}^{q^{i}}, \quad i=0,1, \ldots, m-1, \\
b_{i j}=b_{j i}=a_{j-i}^{q^{i}}+a_{i-j}^{q^{j}}, \quad i \neq j,
\end{gathered}
$$

и индексы у коэффищиентов $a_{s}$ рассматриваются по модулю $m\left(a_{s}=0\right.$, если $s>\lfloor m / 2\rfloor)$. Обозначим через $B=\left[b_{i j}\right]$ симметричную матрицу коэффициентов $b_{i j}$. Тогда симметричная матрица коэффищиентов $C=\left[c_{u v}\right]$ определяется следуюшим образом:

$$
C=D B D^{T}
$$

где

$$
D=\left[\gamma_{i}^{q^{j}}\right]
$$

В силу невырожденности матрицы $D$ (см., например, [2]) ранги матрищ $C$ и $B$ равны: $\operatorname{rank}(C)=\operatorname{rank}(B)$.

Так как $S(\varphi(x))=S_{m}\left(g\left(x_{0}, \ldots, x_{m-1}\right)\right)$ и

$$
\left|S_{m}(g)\right|=q^{m-\frac{1}{2} \operatorname{rank}(C)},
$$

то имеет место следуюшее утверждение.

УТВЕРЖДЕНИЕ 1. Пусть $\varphi(x)$ - произвольный многочлен вида (5) и матрича В определяется соотношениями (6). Тогда

$$
|S(\varphi)|=q^{m-\frac{1}{2} \operatorname{rank}(B)} .
$$

Поскольку мы рассматриваем только нетривиальный случай $\operatorname{rank}(B) \neq 0$, то максимальное значение модуля суммы $S(\varphi)$ получается, когда $\operatorname{rank}(B)=1$, и, следовательно, наша задача сводится к описанию всех многочленов $\varphi(x)$ вида $(5)$ таких, что ранг соответствуюшей матрищы $B$ равен 1 . Случаи четного и нечетного $m$ рассматриваются отдельно. 
I.a. $m=2 k+1, k \geqslant 1$.

TeOpema 1. Пусть

$$
\varphi(x)=a_{0} x^{2}+2 \sum_{s=1}^{k} a_{s} x^{1+q^{s}}
$$

Тогда $|S(\varphi)|=q^{m-\frac{1}{2}}$ (т.е. достигается оценка Вейля), если и только если

$$
a_{s}=a_{0}^{\frac{q^{s}+1}{2}}, \quad s=1, \ldots, k,
$$

где $a_{0}-$ произвольный ненулевой әлемент поля $F_{q^{m}}$. Число различных таких многочленов $\varphi(x)$ равно $q^{m}-1$.

ДокАЗАТЕЛЬСтво. Так как при нечетном $m$ матрица $B$ имеет вид (см. (6))

$$
B=\left[\begin{array}{ccccccccc}
a_{0} & a_{1} & a_{2} & \ldots & a_{k} & a_{k}^{q^{k+1}} & \ldots & a_{2}^{q^{2 k-1}} & a_{1}^{q^{2 k}} \\
a_{1} & a_{0}^{q} & a_{1}^{q} & \ldots & a_{k-1}^{q} & a_{k}^{q} & \ldots & a_{3}^{q^{2 k-1}} & a_{2}^{q^{2 k}} \\
\ldots & \ldots & \ldots & \ldots & \ldots \ldots \ldots & \ldots & \ldots & \ldots & \ldots
\end{array}\right]
$$

то ее ранг равен 1 тогда и только тогда, когда вторая строка пропорциональна первой (любая строка матрицы $B$ является циклическим сдвигом предыдущей строки, каждый элемент которой возведен в степень $q$ ). Поэтому если вторая строка пропорциональна первой, то третья строка пропорциональна второй и т. д. Достаточно проверить, что при заданном $a_{0}$ все остальные коэффициенты $a_{s}(s=1, \ldots, k)$ определяются однозначно в соответствии с (7). Действительно, соотношения (7) следуют непосредственно из пропорциональности подстрок:

$$
\left[\begin{array}{cccccc}
a_{0} & a_{1} & a_{2} & \ldots & a_{k-1} & a_{k} \\
a_{1} & a_{0}^{q} & a_{1}^{q} & \ldots & a_{k-2}^{q} & a_{k-1}^{q}
\end{array}\right]
$$

(сначала находится $a_{1}$, затем $a_{2}$ и т. д.). При этом коэффищиент $a_{1}$ может принимать два значения:

$$
a_{1}= \pm a_{0}^{\frac{q+1}{2}}
$$

а все следуюшие коэффициенты $a_{2}, a_{3}, \ldots, a_{k}$ при заданных $a_{0}$ и $a_{1}$ определяются однозначно. Таким образом, при заданном $a_{0}$ из пропорциональности подстрок следуют два ответа:

$$
a_{s}=a_{0}^{\frac{q^{s}+1}{2}}, \quad s=1,2, \ldots, k,
$$

и

$$
a_{s}=(-1)^{s} a_{0}^{\frac{q^{s}+1}{2}}, \quad s=1,2, \ldots, k .
$$

Однако в силу нечетности $m$ все остальные пропорции вьполнены лишь при первом ответе. Так как $a_{0}$ может принимать любое ненулевое значение из поля $F_{Q}$, а остальные $a_{s}, s=1, \ldots, k$, определяются однозначно при заданном $a_{0}$, то число различных многочленов вида (5) с максимальным модулем тригонометрической суммы равно $q^{m}-1$. 
Теорема 1 наводит на мысль, что сушествуют многочлены вида (5) меньшей степени, для которых достигается оценка Вейля. Действительно, рассмотрим такие многочлены, у которых все коэффициенты, начиная с $(r+1)$-го, равны нулю $\left(a_{r+1}=\cdots=\right.$ $\left.a_{\lfloor m / 2\rfloor}=0\right)$. Оценка Вейля для таких многочленов равна $q^{r+k+\frac{1}{2}}$ и она достигается тогда и только тогда, когда ранг соответствующей матрищы $B$ (см. утверждение 1$)$ равен $2(k-r)+1$. В теореме 1 мы изучили случай, когда $r=k$ и даже описали все такие матрицы. Обший случай довольно труден для решения, но другой крайний случай, когда $r=1$ (случай $r=0$ хорошо известен как суммы Гаусса), мы сейчас опишем, но при условии, что коэффициенты $a_{0}, a_{1}$ выбираются из поля $F_{q}$. В этом случае матрица $B$ имеет вид

$$
B=\left[\begin{array}{cccccc}
a_{0} & a_{1} & 0 & \ldots & 0 & a_{1} \\
a_{1} & a_{0} & a_{1} & \ldots & 0 & 0 \\
0 & a_{1} & a_{0} & \ldots & 0 & 0 \\
\ldots & \ldots & \ldots & \ldots & \ldots & \ldots \\
0 & 0 & 0 & \ldots & a_{0} & a_{1} \\
a_{1} & 0 & 0 & \ldots & a_{1} & a_{0}
\end{array}\right] .
$$

Вычисления показьвают, что ранг этой матрицы равен $2 k-1$ тогда и только тогда, когда равен нулю определитель $\Delta_{k}=\Delta_{k}\left(a_{0}, a_{1}\right)$ следующей матрищы размера $k \times k$ :

$$
\left[\begin{array}{cccccc}
a_{0} & a_{1} & 0 & \ldots & 0 & 0 \\
a_{1} & a_{0} & a_{1} & \ldots & 0 & 0 \\
0 & a_{1} & a_{0} & \ldots & 0 & 0 \\
\ldots & \ldots & \ldots & \ldots & \ldots & \ldots \ldots \\
0 & 0 & 0 & \ldots & a_{0} & a_{1} \\
0 & 0 & 0 & \ldots & a_{1} & a_{0}-a_{1}
\end{array}\right] .
$$

Так как

$$
\Delta_{k}=a_{0} \Delta_{k-1}-a_{1}^{2} \Delta_{k-2}, \quad k \geqslant 2, \quad \Delta_{0}=1, \quad \Delta_{1}=a_{0}-a_{1},
$$

то

$$
\Delta_{k}\left(a_{0}, a_{1}\right)=\sum_{j=0}^{k}(-1)^{\lceil j / 2\rceil}\left(\begin{array}{c}
k-\lceil j / 2\rceil \\
\lfloor j / 2\rfloor
\end{array}\right) a_{0}^{k-j} a_{1}^{j} .
$$

Поэтому имеет место следуюший результат.

Teopema 2. Пусть $\varphi(x)=a_{0} x^{2}+2 a_{1} x^{1+q}$, әде $a_{0}, a_{1} \in F_{q}$ u $a_{1} \neq 0$. Тогдa

$$
|S(\varphi)|=q^{k+\frac{3}{2}}
$$

(т.е. достигается оченка Вейля) тогда и только тогда, когда

$$
\Delta_{k}\left(a_{0}, a_{1}\right)=0 .
$$

Нетрудно видеть, что уравнение $\Delta_{k}\left(a_{0}, a_{1}\right)=0$ не всегда имеет решение $a_{0}, a_{1} \in F_{q}^{*}$. Например, уравнение

$$
\Delta_{2}\left(a_{0}, a_{1}\right)=a_{0}^{2}-a_{0} a_{1}-a_{1}^{2}=0
$$


не имеет ненулевых решений в поле $F_{3}$. Однако это уравнение всегда имеет решение, например, в любом четном расширении поля $F_{3}$ или в любом поле характеристики 5 .

Но при $k=1$ уравнение $\Delta_{1}\left(a_{0}, a_{1}\right)=a_{0}-a_{1}=0$ всегда имеет ненулевое решение $a_{0}=a_{1}$ в $F_{q}$. Этот пример был приведен Степановьм [4] (впрочем, он следует и из теоремы 1). Отсюда и из замечания Родье [5] о том, что многочлен над конечньп полем, достигающий границы Вейля, остается таковым и над любьм расширением этого поля, вытекает, что пара $a_{0}=a_{1}$ является решением уравнения $\Delta_{k}\left(a_{0}, a_{1}\right)=0$ при любом $k$ таком, что 3 делит $2 k+1$ (возможны также и другие решения).

I.b. $m=2 k, k \geqslant 1$.

TEOpema 3. Пусть

$$
\varphi(x)=a_{0} x^{2}+2 \sum_{s=1}^{k} a_{s} x^{1+q^{s}} .
$$

Тогда

$$
|S(\varphi)|=q^{m-\frac{1}{2}}
$$

если и только если либо

$$
a_{s}=a_{0}^{\frac{q^{s}+1}{2}}, \quad s=1, \ldots, k-1,
$$

где $a_{0}-$ произвольный квадратичный выцет поля $F_{q^{2 k}}$, а $a_{k}-$ произвольньй корень уравнения

$$
a_{k}+a_{k}^{q^{k}}=a_{0}^{\frac{q^{k}+1}{2}}
$$

либо

$$
a_{s}=(-1)^{s} a_{0}^{\frac{q^{s}+1}{2}}, \quad s=1, \ldots, k-1,
$$

где $a_{0}$ - произвольный квадратичный вычет поля $F_{q^{2 k}}$, а $a_{k}-$ произвольный корень уравнения

$$
a_{k}+a_{k}^{q^{k}}=(-1)^{k} a_{0}^{\frac{q^{k}+1}{2}} .
$$

Число таких различных многочленов равно $\left(q^{m}-1\right) q^{m / 2}$.

ДокАЗАТЕЛЬСтво. При четном $m$ матрица $B$ имеет вид (см. (6)):

$$
D=\left[\begin{array}{ccccccccc}
a_{0} & a_{1} & a_{2} & \ldots & a_{k-1} & a_{k}+a_{k}^{q^{k}} & a_{k-1}^{q^{k+1}} & \ldots & a_{1}^{q^{2 k-1}} \\
a_{1} & a_{0}^{q} & a_{1}^{q} & \ldots & a_{k-2}^{q} & a_{k-1}^{q} & a_{k}^{q}+a_{k}^{q^{k+1}} & \ldots & a_{2}^{q^{2 k-1}} \\
\ldots \ldots & \ldots & \ldots & \ldots \ldots \ldots \ldots \ldots \ldots \ldots \ldots \ldots \ldots \ldots \ldots \ldots \ldots \ldots \ldots \ldots
\end{array}\right]
$$

Как и в предыдушем случае, ее ранг равен 1 тогда и только тогда, когда вторая строка пропорциональна первой. Вновь, как и в теореме 1 , задав произвольно коэффициент $a_{0}$, остальные коэффищиенты найдем из условия пропорциональности подстрок

$$
\left[\begin{array}{cccccc}
a_{0} & a_{1} & a_{2} & \ldots & a_{k-1} & a_{k}+a_{k}^{q^{k}} \\
a_{1} & a_{0}^{q} & a_{1}^{q} & \ldots & a_{k-2}^{q} & a_{k-1}^{q}
\end{array}\right]
$$


Ситуация, как мы видим, совпадает с ситуацией теоремы 1 , за исключением последнего коэффициента $a_{k}$, для которого мы имеем следуюшее уравнение:

$$
a_{k}+a_{k}^{q^{k}}=a_{0}^{\frac{q^{k}+1}{2}}, \text { когда } a_{1}=a_{0}^{\frac{q+1}{2}},
$$

и

$$
a_{k}+a_{k}^{q^{k}}=(-1)^{k} a_{0}^{\frac{q^{k}+1}{2}}, \text { когда } a_{1}=-a_{0}^{\frac{q+1}{2}} .
$$

Нетрудно видеть, что любое из этих уравнений имеет решение тогда и только тогда, когда $a_{0}$ является квадратичньм вычетом в поле $F_{q^{2 k}}$, и что число решений равно $q^{k}$. Несложно проверить, что при таком задании коэффициентов $a_{0}, a_{1}, \ldots, a_{k}$ остальные пропорции также вьполнены. Общее число различных решений $a_{0}, a_{1}, \ldots, a_{k}$ равно

$$
\frac{q^{2 k}-1}{2} \cdot 2 \cdot q^{k}
$$

(так как $a_{0}$ - произвольньй квадратичньй вычет; $a_{1}$ принимает точно два значения при заданном $a_{0}$; каждое $a_{i}, 1<i<k$, находится однозначно при заданных $a_{0}$ и $a_{1}$; а $a_{k}$ принимает точно $q^{k}$ значений при заданных $a_{0}$ и $\left.a_{1}\right)$.

ЗАмечАниЕ 1 . Частным случаем теорем 1 и 3 является теорема 1 работы [4].

Как мы видим, в теореме 3 не достигается оценка Вейля. Однако вновь, как и в предыдушем случае, мы можем рассмотреть многочлены вида (5) меньшей степени и показать, что по крайней мере для некоторых из них граница Вейля достигается. Рассмотрим сначала многочлены, у которых $a_{k}=0$. Оценка Вейля для таких многочленов равна $q^{2 k-1}$. Согласно утверждению 1 она достигается на многочленах, для которых соответствуюшая матрица $B$ имеет ранг 2. Вновь рассматривая многочлены с коэффициентами только из маленького поля $F_{q}$, несложно доказать следующую теорему.

Teopema 4. Пyсmb

$$
\varphi(x)=a_{0} x^{2}+2 \sum_{s=1}^{k-1} a_{s} x^{1+q^{s}},
$$

где $a_{s} \in F_{q}, s=0,1, \ldots, k-1$. Тогда

$$
|S(\varphi)|=q^{2 k-1}
$$

(т.е. достигается оченка Вейля) тогда и только тогда, когда

$$
a_{s}=0 \text { для } s \equiv k(\bmod 2) \quad u \quad a_{s}=a \partial \Omega_{я} s \equiv k-1(\bmod 2),
$$

где а - произвольный ненулевой әлемент $F_{q}$.

Другой крайний случай - когда все коэффициенты, начиная с $a_{2}$, равны нулю. Здесь мы имеем теорему, аналогичную теореме 2 . Обозначим через $\Omega_{k}\left(a_{0}, a_{1}\right)$ определитель матришы размера $k \times k$, отличаюшейся от матрицы (8) лишь элементом в нижнем правом углу, где вместо элемента $a_{0}-a_{1}$ стоит элемент $a_{0}$. Нетрудно вычислить, что

$$
\Omega_{k}\left(a_{0}, a_{1}\right)=\sum_{j=0}^{k}(-1)^{j}\left(\begin{array}{c}
k-j \\
j
\end{array}\right) a_{0}^{k-2 j} a_{1}^{2 j} .
$$

Поэтому имеет место следуюший результат. 
Tеорема 5. Пусть $\varphi(x)=a_{0} x^{2}+2 a_{1} x^{1+q}$, где $a_{0}, a_{1} \in F_{q}$ u $a_{1} \neq 0$. Тогда

$$
|S(\varphi)|=q^{k+1}
$$

(т.е. достигается оченка Вейля) тогда и только тогда, когда

$$
\Omega_{k}\left(a_{0}, a_{1}\right)=0
$$

ЗАмЕчАНИЕ 2. Отметим, что при нечетных $k$ любая пара $a_{0}=0, a_{1} \neq 0$ является решением уравнения $\Omega_{k}\left(a_{0}, a_{1}\right)=0$.

ЗАмечАниЕ 3. В работе [6] Вольфманн, в частности, вычислил точное значение тригонометрической суммы одночлена $a_{r} x^{1+q^{r}}$, где $r$ делит $k, a_{r} \in F_{Q}{ }^{*}$, и дал необходимое и достаточное условие, чтобы эта сумма достигала оценки Вейля. Это условие можно вывести и из рассмотрения матрищы $B$, имеющей достаточно простой вид в случае одночленов.

Заключительное замечание относится к произвольному $m$ - как четному, так и нечетному, и дает еше один пример многочленов, для которых достигается гранища Вейля. Рассмотрим многочлен

$$
f_{a}(x)=a \sum_{s \leqslant r: s \equiv r(\bmod m-2 r)} x^{1+q^{s}}, \quad a \in F_{q^{m-2 r}}^{*} .
$$

Нетрудно проверить, что ранг матрицы $B$, соответствующей этому многочлену, равен $m-2 r$, если $m-2 r$ делит $m$ (в этом случае матрица $B$ содержит ровно $m-2 r$ различных строк). Поэтому имеет место следующее утверждение.

УТВЕРЖДЕНИЕ 2. Пусть $m-2 r$ делит $m$. Тогда для многочлена $f_{a}(x)$ достигается гранища Вейля:

$$
\left|S\left(f_{a}\right)\right|=q^{r+\frac{m}{2}}
$$

\section{II. Случай четной характеристики $(p=2)$}

В этом случае максимальное значение модуля тригонометрической суммы для многочленов вида (3) равно $q^{m-1}$ (см. [1]). С.А. Степанов [4] дал примеры таких многочленов в явном виде при нечетных $m$. Мы приводим здесь широкий класс таких многочленов как для нечетного, так и для четного $m$, но не утверждаем, что описьваем все такие многочлены (как в случае нечетного $q$ ), хотя и полагаем, что это так.

В случае четной характеристики нет аналога утверждения 1 , что вьнуждает нас идти другим путем. Известно [2], [3], что только две квадратичные формы от $m$ переменных в приведенном виде над полем $F_{q}$ имеют модуль тригонометрической суммы, равный $q^{m-1}$ :

$$
z_{1}^{2}+z_{1} z_{2}+a z_{2}^{2}, \quad \operatorname{Tr}_{q}(a)=1
$$


Поэтому наша задача заключается в нахождении таких многочленов вида (3), что соответствуюшие им квадратичные формы (см. (4))

$$
g\left(x_{0}, x_{1}, \ldots, x_{m-1}\right)=\operatorname{Tr}_{Q \rightarrow q}\left(f\left(\sum_{s=0}^{m-1} x_{s} \gamma_{s}\right)\right)=\sum_{0 \leqslant u \leqslant v \leqslant m-1} c_{u v} x_{u} x_{v}
$$

приводятся к одному из указанных видов. По аналогии с п. І вьпишем вначале

$$
h(x)=\operatorname{Tr}_{Q \rightarrow q}\left(\sum_{s=0}^{k} a_{s} x^{1+q^{s}}\right)=\sum_{0 \leqslant i \leqslant j \leqslant m-1} b_{i j} x^{q^{i}+q^{j}} .
$$

Однако уже для вычисления коэффициентов $b_{i j}$ через $a_{s}$ удобнее случаи нечетного и четного $m$ рассматривать отдельно.

II.a. $m=2 k+1, k \geqslant 1$. В этом случае коэффициенты $b_{i j}$ вычисляются следующим образом:

$$
b_{i j}= \begin{cases}a_{0}^{q^{i}}, & \text { когда } i=j, \\ a_{j-i}^{q^{i}}, & \text { когда } 0 \leqslant i<j \leqslant k+i, \\ a_{m+i-j}^{q^{j}}, & \text { когда } k+i<j \leqslant m-1 .\end{cases}
$$

Сначала рассмотрим ситуацию, когда квадратичная форма (11) может быть приведена к виду (9). Положим

$$
z_{1}(x)=\sum_{i=0}^{m-1} \alpha_{i} x^{q^{i}}, \quad z_{2}(x)=\sum_{j=0}^{m-1} \beta_{j} x^{q^{j}}
$$

и потребуем вьполнения следующего равенства:

$$
h(x)=z_{1}(x) z_{2}(x) .
$$

Из (12)-(15) получаем следующую систему соотношений:

$$
\left\{\begin{aligned}
a_{0}^{q^{i}} & =\alpha_{i} \beta_{i}, & & \text { когда } i=0,1, \ldots, m-1, \\
a_{j-i}^{q^{i}} & =\alpha_{i} \beta_{j}+\alpha_{j} \beta_{i}, & & \text { когда } 0 \leqslant i<j \leqslant k+i, \\
a_{m+i-j}^{q^{j}} & =\alpha_{i} \beta_{j}+\alpha_{j} \beta_{i}, & & \text { когда } k+i<j \leqslant m-1 .
\end{aligned}\right.
$$

Пусть $\alpha_{i}$ и $\beta_{j}$ удовлетворяют следующему дополнительному ограничению:

$$
\alpha_{i}=\alpha_{0}^{q^{i}}, \quad \beta_{j}=\beta_{0}^{q^{j}}, \quad \text { когда } i, j=1, \ldots, m-1 .
$$

При этом система соотношений (16) переходит в систему:

$$
\begin{aligned}
& a_{0}=\alpha_{0} \beta_{0}, \\
& a_{s}=\alpha_{0} \beta_{0}^{q^{s}}+\alpha_{0}^{q^{s}} \beta_{0}, \quad s=1, \ldots, k .
\end{aligned}
$$


При условии $(17) z_{1}(x)$ и $z_{2}(x)$ из (14) могут рассматриваться как линейные формы над полем $F_{q}$ от $m$ переменных $x_{0}, \ldots, x_{m-1}$. Действительно, подставляя выражение $x$ через базис $\gamma_{0}, \ldots, \gamma_{m-1}$, получаем

$$
z_{1}\left(x_{0}, \ldots, x_{m-1}\right)=\sum_{i=0}^{m-1} \alpha_{0}^{q^{i}}\left(\sum_{s=0}^{m-1} \gamma_{s} x_{s}\right)^{q^{i}}=\sum_{s=0}^{m-1} \operatorname{Tr}_{Q \rightarrow q}\left(\alpha_{0} \gamma_{s}\right) x_{s}
$$

и

$$
z_{2}\left(x_{0}, \ldots, x_{m-1}\right)=\sum_{i=0}^{m-1} \beta_{0}^{q^{i}}\left(\sum_{s=0}^{m-1} \gamma_{s} x_{s}\right)^{q^{i}}=\sum_{s=0}^{m-1} \operatorname{Tr}_{Q \rightarrow q}\left(\beta_{0} \gamma_{s}\right) x_{s}
$$

Линейные формы $z_{1}\left(x_{0}, \ldots, x_{m-1}\right)$ и $z_{2}\left(x_{0}, \ldots, x_{m-1}\right)$ независимы над $F_{q}$ тогда и только тогда, когда элементы $\alpha_{0}$ и $\beta_{0}$ поля $F_{Q}$ линейно независимы над полем $F_{q}$. Следовательно, при любом таком выборе $\alpha_{0}$ и $\beta_{0}$

$$
\alpha_{0}, \beta_{0} \in F_{Q}^{*}, \quad \alpha_{0} \neq c \beta_{0}, \quad c \in F_{q}^{*},
$$

и выборе коэффициентов $a_{0}, a_{1}, \ldots, a_{k}$ многочлена $f(x)$ вида $(3)$, удовлетворяюшем условию (18), соответствуюшая квадратичная форма (см. (11)), приводится к виду (9):

$$
g\left(x_{0}, \ldots, x_{m-1}\right)=z_{1}\left(x_{0}, \ldots, x_{m-1}\right) z_{2}\left(x_{0}, \ldots, x_{m-1}\right) .
$$

Оценим снизу число таких различных многочленов $f(x)$ вида (3). Ясно, что число различных упорядоченных пар $\left(\alpha_{0}, \beta_{0}\right)$, удовлетворяюших условию (19), равно $\left(q^{m}-1\right)\left(q^{m}-q\right)$. С другой стороны, число различных пар $\left(\alpha_{0}, \beta_{0}\right)$, задаюших один и тот же набор двух первых коэффициентов $a_{0}$ и $a_{1}$ (см. (18)):

$$
\begin{gathered}
\alpha_{0} \beta_{0}=a_{0}, \\
\alpha_{0} \beta_{0}^{q}+\alpha_{0}^{q} \beta_{0}=a_{1},
\end{gathered}
$$

не превосходит 2(q-1). Поэтому число таких различных многочленов вида (3) с максимальным модулем тригонометрической суммы $q^{m-1}$ не меньше, чем $\left(q^{m}-1\right)\left(q^{m}-q\right) / 2(q-1)$ (на самом деле, равно, так как наряду с парой $\left(\alpha_{0}, \beta_{0}\right)$ тот же самьй набор коэффициентов $a_{0}, a_{1}, \ldots, a_{k}$ дают пары $\left(\beta_{0}, \alpha_{0}\right)$ и $\left(c \alpha_{0}, c^{-1} \beta_{0}\right)$, где $c$ - произвольный ненулевой элемент поля $F_{q}$ ).

Перейдем теперь к ситуации, когда квадратичная форма (11) может быть приведена к виду (10). Рассуждая так же, как и в предыдушем случае, получим, что единственным отличием является замена системы соотношений (18) на другую:

$$
\begin{aligned}
& a_{0}=\alpha_{0}^{2}+\alpha_{0} \beta_{0}+a \beta_{0}^{2}, \\
& a_{s}=\alpha_{0} \beta_{0}^{q^{s}}+\alpha_{0}^{q^{s}} \beta_{0}, \quad s=1, \ldots, k,
\end{aligned}
$$

где, конечно, $\alpha_{0}$ и $\beta_{0}$ удовлетворяют условию (19). Оценим снизу число различных наборов коэффициентов $a_{0}, a_{1}, \ldots, a_{k}$, задаваемых условием (20). Так как число различных пар $\left(\alpha_{0}, \beta_{0}\right)$, задающих один и тот же набор двух первых коэффициентов $a_{0}$ и $a_{1}(\mathrm{~cm} .(20))$ :

$$
\begin{aligned}
& a_{0}=\alpha_{0}^{2}+\alpha_{0} \beta_{0}+a \beta_{0}^{2}, \\
& a_{1}=\alpha_{0} \beta_{0}^{q}+\alpha_{0}^{q} \beta_{0},
\end{aligned}
$$


не превосходит $2(q+1)$ (по теореме Безу), то число таких различных наборов не меньше, чем $\left(q^{m}-1\right)\left(q^{m}-q\right) / 2(q+1)$ - на самом деле, равно, так как наряду с парой $\left(\alpha_{0}, \beta_{0}\right)$ тот же самый набор коэффициентов $a_{0}, a_{1}, \ldots, a_{k}$ дают пары $\left(c_{11} \alpha_{0}+c_{12} \beta_{0}, c_{21} \alpha_{0}+c_{22} \beta_{0}\right)$, где $c_{i j} \in F_{q}$ удовлетворяют следуюшей системе уравнений:

$$
\begin{aligned}
& c_{11}^{2}+c_{11} c_{21}+a c_{21}^{2}=1, \\
& c_{11} c_{22}+c_{12} c_{21}=1, \\
& c_{12}^{2}+c_{12} c_{22}+a c_{22}^{2}=a,
\end{aligned}
$$

число решений которой равно $2(q+1)$.

Нетрудно показать, что, выбирая другой элемент $a^{\prime} \neq a, a^{\prime} \in F_{q}, \operatorname{Tr}_{q}\left(a^{\prime}\right)=1$, в квадратичной форме вида (10), мы получим тот же самый набор коэффициентов $\left(a_{0}, a_{1}, \ldots, a_{k}\right)$, что и при элементе $a$. Действительно, рассмотрим две системы уравнений с двумя неизвестными

$$
\begin{aligned}
& x^{2}+x y+a y^{2}=a_{0}, \\
& x y^{q^{s}}+x^{q^{s}} y=a_{s}, \quad s=1, \ldots, k
\end{aligned}
$$

и

$$
\begin{aligned}
& u^{2}+u v+a^{\prime} v^{2}=a_{0}, \\
& u v^{q^{s}}+u^{q^{s}} v=a_{s}, \quad s=1, \ldots, k
\end{aligned}
$$

Пусть $(x, y)$ - решение системы (21). Тогда $u=b_{1} x+a^{1 / 2} y, v=a^{-1 / 2} x$ является решением системы $(22)$, где элемент $b_{1} \in F_{q}$ удовлетворяет уравнению

$$
b_{1}^{2}+b_{1} a^{-1 / 2}+a^{\prime} a^{-1}=1
$$

(это уравнение всегда имеет решение, так как $\operatorname{Tr}_{q}\left(a+a^{\prime}\right)=0$ ).

С другой стороны, даже множества пар $\left(a_{0}, a_{1}\right)$ первых коэффициентов, задаваемых квадратичной формой (9) и квадратичной формой (10) с фиксированньм $a$, не пересекаются. Для доказательства этого достаточно показать, что две системы уравнений

$$
\begin{aligned}
& u v=a_{0}, \\
& u v^{q}+u^{q} v=a_{1}
\end{aligned}
$$

и

$$
\begin{aligned}
& x^{2}+x y+a y^{2}=a_{0} \\
& x y^{q}+x^{q} y=a_{1}
\end{aligned}
$$

не имеют одновременно решений в поле $F_{Q}$ при фиксированных $a_{0}, a_{1} \in F_{Q}^{*}$. Действительно, если $(x, y)$ - решение системы $(24)$, то система (23) не имеет решения. Предположим противное. Рассмотрим эквивалентную ей систему

$$
\begin{aligned}
& u v=a_{0}, \\
& v^{q-1}+u^{q-1}=a_{1} / a_{0} .
\end{aligned}
$$


Если эта система разрешима, то в поле $F_{Q}$ разрешимо квадратное уравнение $\left(z=v^{q-1}\right)$ :

$$
z^{2}+\frac{a_{1}}{a_{0}} z+a_{0}^{q-1}=0 .
$$

Но это квадратное уравнение имеет решение в поле $F_{Q}$ тогда и только тогда, когда

$$
\operatorname{Tr}_{Q}\left(\frac{a_{0}^{q-1}}{\left(a_{1} / a_{0}\right)^{2}}\right)=0
$$

В силу (24) условие (25) эквивалентно следуюшему:

$$
\begin{aligned}
& \operatorname{Tr}_{Q}\left(\frac{\left(x^{2 q}+x^{q} y^{q}+a y^{2 q}\right)\left(x^{2}+x y+a y^{2}\right)}{x^{2} y^{2 q}+x^{2 q} y^{2}}\right) \\
& \quad=\operatorname{Tr}_{Q}(a)+\operatorname{Tr}_{Q}\left(\frac{x^{2 q+2}+x^{2 q+1} y+x^{q+2} y^{q}+x^{q+1} y^{q+1}}{x^{2} y^{2 q}+x^{2 q} y^{2}}\right) \\
& \quad=\operatorname{Tr}_{Q}(a)=\operatorname{Tr}_{q}(a)=0,
\end{aligned}
$$

что противоречит условию (10).

Поэтому общее число построенных нами различных многочленов вида (3) с максимальным модулем тригонометрической суммы $q^{m-1}$ равно

$$
\left(q^{m}-1\right)\left(q^{m}-q\right)\left(\frac{1}{2(q-1)}+\frac{1}{2(q+1)}\right)=\frac{\left(q^{m}-1\right)\left(q^{m-1}-1\right) q^{2}}{q^{2}-1} .
$$

Тем самьм мы доказали следуюшую теорему.

Теорема 6. Пусть $f(x)=\sum_{s=0}^{k} a_{s} x^{1+q^{s}}$. Если коэффициенты $a_{s}$ удовлетворяют условиям (18) или (20), где $\operatorname{Tr}_{q}(a)=1, a \in F_{q}$, а әлементы $\alpha_{0}, \beta_{0}$ удовлетворяют условию (19), то

$$
|S(f)|=q^{m-1} .
$$

Число таких различных многочленов равно

$$
\frac{\left(q^{m}-1\right)\left(q^{m-1}-1\right) q^{2}}{q^{2}-1} .
$$

ЗАмечАниЕ 4. Частным случаем теоремы 6 является теорема 4 работы [4].

II.b. $m=2 k, k \geqslant 1$. В этом случае коэффициенты $b_{i j}$ в (12) вычисляются следуюшим образом:

$$
b_{i j}= \begin{cases}a_{0}^{q^{i}}, & \text { когда } i=j, \\ a_{j-i}^{q^{i}}, & \text { когда } 0 \leqslant i<j<k+i, \\ a_{k}^{q^{i}}+a_{k}^{q^{j}}, & \text { когда } j=k+i, \\ a_{m+i-j}^{q^{j}}, & \text { когда } k+i<j \leqslant m-1 .\end{cases}
$$


Те же рассуждения, что и в п. II.а, с учетом (26) вместо (13), приводят к следующим двум системам соотношений между коэффициентами $a_{0}, \ldots, a_{k}$ и параметрами $\alpha_{0}, \beta_{0}$, аналогичным системам (18) и (20):

$$
\begin{aligned}
a_{0} & =\alpha_{0} \beta_{0}, \\
a_{s} & =\alpha_{0} \beta_{0}^{q^{s}}+\alpha_{0}^{q^{s}} \beta_{0}, \quad s=1, \ldots, k-1, \\
a_{k}+a_{k}^{q^{k}} & =\alpha_{0} \beta_{0}^{q^{k}}+\alpha_{0}^{q^{k}} \beta_{0}
\end{aligned}
$$

и

$$
\begin{aligned}
a_{0} & =\alpha_{0}^{2}+\alpha_{0} \beta_{0}+a \beta_{0}^{2}, \\
a_{s} & =\alpha_{0} \beta_{0}^{q^{s}}+\alpha_{0}^{q^{s}} \beta_{0}, \quad s=1, \ldots, k-1, \\
a_{k}+a_{k}^{q^{k}} & =\alpha_{0} \beta_{0}^{q^{k}}+\alpha_{0}^{q^{k}} \beta_{0} .
\end{aligned}
$$

Системы (27) и (28) отличаются от аналогичных систем (18) и (20) только последним соотношением, связывающим коэффициент $a_{k}$ с параметрами $\alpha_{0}, \beta_{0}$. Поэтому каждой паре параметров $\left(\alpha_{0}, \beta_{0}\right)$ соответствует не один набор коэффициентов $a_{0}, \ldots, a_{k}$, как в предыдущем случае, а $q^{k}$ наборов $a_{0}, \ldots, a_{k-1}, a_{k}$, отличающихся только последним коэффициентом $a_{k}$. Действительно, при фиксированных $\alpha_{0}, \beta_{0}$ уравнение относительно $a_{k}$

$$
a_{k}+a_{k}^{q^{k}}=\alpha_{0} \beta_{0}^{q^{k}}+\alpha_{0}^{q^{k}} \beta_{0}
$$

имеет точно $q^{k}$ различных решений в поле $F_{Q}$. Следовательно, общее число различных наборов коэффициентов $a_{0}, a_{1}, \ldots, a_{k}$ при четном $m$ в $q^{m / 2}$ раз больше, чем в случае нечетного $m$. За исключением указанного единственного отличия, случаи четного и нечетного $m$ полностью аналогичны. Поэтому имеет место следуюший результат.

ТЕОРема 7. Пусть $f(x)=\sum_{s=0}^{k} a_{s} x^{1+q^{s}}$. Если все коэффициенты $a_{s}$ удовлетворяют условиям (27) или (28), где $\operatorname{Tr}_{q}(a)=1, a \in F_{q}$, а әлементы $\alpha_{0}, \beta_{0}$ удовлетворяют условию (19), то $|S(f)|=q^{m-1}$. Число таких различных многочленов равно

$$
\frac{\left(q^{m}-1\right)\left(q^{m-1}-1\right) q^{m / 2} q^{2}}{q^{2}-1} .
$$

При $k \geqslant 2$ всегда сушествуют элементы $\alpha_{0}, \beta_{0}$ такие, что $a_{k}=0\left(\alpha_{0} / \beta_{0} \in F_{q^{k}}\right)$. Для таких многочленов достигается оценка Вейля. Поэтому имеет место следующее утверждение.

СлЕДСТВИЕ 1. Среди указанных в теореме 7 многочленов при $k \geqslant 2$ имеются многочлены степени $q^{k-1}+1$ (т.е. многочлены с $\left.a_{k}=0\right)$. На таких многочленах достигается оценка Вейля.

Из этого следствия, в частности, вытекает следующее равенство:

$$
S\left(q^{k}+1, q^{2 k+1}\right)=q^{2 k+\frac{1}{2}},
$$

где $k \geqslant 1$ и $q$-четная степень 2 , которое в [1] мы умели доказьвать для нечетных $q$; здесь $S(d, Q)=\max |S(f)|$, где максимум берется по всем многочленам над $F_{Q}$ степени не выше $d$ таким, что $\operatorname{Tr}_{Q}(f(x)) \neq$ const. В частности, при $q=4$ и $k=1$ это равенство имеется у В. И. Левенштейна [7]. Мы по-прежнему полагаем [1], что когда $q$-нечетная степень 2, то равенство (29) не имеет места. 
ПРЕДПОЛОЖЕНИЕ 1. Пусть $q=2^{l}$, где $l$ - нечетное. Тогда

$$
S\left(q^{k}+1, q^{2 k+1}\right)=q^{2 k}
$$

Толчком к написанию данной работы послужило обсуждение доклада авторов на семинаре А. А. Карацубы по теории чисел Московского государственного университета, за что авторы признательны участникам семинара.

\section{СПИСОК ЛИТЕРАТУРЫ}

[1] Бассалыго Л. А., Зиновьев В. А., Лицын С. М. Нижняя оценка полных тригонометрических сумм в терминах кратных сумм // Докл. АН СССР. 1988. Т. 300. № 5. С. 1033-1036.

[2] Лидл Р., Нидеррайтер Г. Конечные поля. М.: Мир, 1988.

[3] Малышев А. В. О представлении целых чисел положительными квадратичными формами // Труды МИАН. 1962. Т. 65.

[4] Степанов С.А. О нижних оценках сумм характеров над конечными полями // Дискретн. матем. 1991. Т. 3. № 2. С. 77-86.

[5] Rodier F. Estimation asymptotique de la distance minimale du dual des codes BCH et polynomes de Dickson // Discrete Math. 1996. V. 149. P. 205-221.

[6] Wolfmann J. The number of points on certain algebraic curves over finite fields // Comm. Algebra. 1989. V. 17. № 8. P. 2055-2060.

[7] Л евенштейн В. И. Границы для упаковок метрических пространств и некоторые их приложения // Проблемы кибернетики. 1983. Т. 40. С. 43-110. 